\title{
Time to stop using hepatic arterial infusion chemotherapy (HAIC) for advanced hepatocellular carcinoma? - the SCOOP-2 trial experience
}

\author{
Markus Peck-Radosavljevic^, Simona Bota, Florian Hucke \\ Innere Medizin und Gastroenterologie (IMuG) mit Zentraler Aufnahme- und Erstversorgung (ZAE), Klinikum Klagenfurt am Wörthersee, \\ Klagenfurt, Austria \\ Correspondence to: Markus Peck-Radosavljevic. Prim., Univ., Prof., Dr., Innere Medizin und Gastroenterologie (IMuG) mit Zentraler Aufnahme- und \\ Erstversorgung (ZAE), Klinikum Klagenfurt am Wörthersee, Feschnigstrasse 11, 9020 Klagenfurt, Austria. Email: markus@peck.at. \\ Comment on: Kondo M, Morimoto M, Kobayashi S, et al. Randomized, phase II trial of sequential hepatic arterial infusion chemotherapy and \\ sorafenib versus sorafenib alone as initial therapy for advanced hepatocellular carcinoma: SCOOP-2 trial. BMC Cancer 2019;19:954.
}

Submitted Apr 23, 2020. Accepted for publication May 01, 2020.

doi: $10.21037 /$ atm-2020-96

View this article at: http://dx.doi.org/10.21037/atm-2020-96

Hepatic arterial infusion chemotherapy (HAIC) is one of several treatment modalities of unproven benefit for advanced stage hepatocellular carcinoma (HCC) that cannot be found in international guidelines $(1,2)$ but retain a certain popularity with a few specialists [others include selective internal radiotherapy (SIRT), conventional chemotherapy with cytotoxic drugs, monotherapy with immune-oncologic drugs, etc.]. HAIC remains a common practice in particular in several Asian countries (3), while it never was able to attract much interest in Western medical practice. The main reason is lack of a proven benefit from randomized controlled trials (RCTs) neither against placebo nor against an established active control treatment (4). Most of the RCTs on HAIC compare one unproven regimen with another unproven regimen, which mostly gives information about the toxicity but not about efficacy of certain treatment combinations (5). At the same time, the use of HAIC dates back to before tyrosine multikinase inhibitors (TKIs) were shown to improve survival in patients with HCC and were mostly used for lack of alternative treatment for these patients and the observation that intraarterial infusion of chemotherapeutic agents seemed to be less toxic than systemic intravenous administration.

When sorafenib became standard of care for advanced stage HCC and the only systemic treatment at that time with a proven survival benefit, it became clear that HAIC would not offer any efficacy benefit compared to sorafenib. Researchers argued in favor of conducting combination trials of HAIC with TKIs in an effort to potentially improve the outcome of TKI-treatment alone for advanced stage HCC (5). It seems likely that these considerations were also fueled by the initial results from combining sorafenib with systemic chemotherapy with doxorubicin in advanced stage HCC-patients (6). But looking more closely at the survival data in the randomized phase- 2 trial of the doxorubicinsorafenib combination, it becomes clear that the difference in survival between the two groups more likely comes from the reduced survival in the doxorubicin monotherapy group [median overall survival (OS): 6.5 months], which was markedly shorter compared to the survival in the control group without therapy in the SHARP-trial (7). As confirmation, the follow-up randomized phase- 3 trial could not show any benefit of adding doxorubicin to sorafenib in advanced stage HCC, compared to sorafenib treatment alone (8), indicating that all the survival benefit was attributable to sorafenib treatment only.

Kondo et al. now report on the SCOOP-2 trial, a phase-2 RCT of HAIC followed by Sorafenib compared to

^ORCID-ID: 0000-0002-0597-2728. 
standard Sorafenib monotherapy in patients with advanced stage HCC (9). The authors of this multicenter study from Japan randomized 70 patients with HCC BCLC B/C not suitable for locoregional therapy to receive either HAIC with cisplatin until progression (median two cycles), followed by standard-dose sorafenib or standarddose sorafenib alone. Sorafenib was continued until progression or beyond, depending on physician decision. Median treatment duration was similar and short (2.6 vs. 2.7 months) in both groups and the median daily dose of sorafenib in both groups was $400 \mathrm{mg}$ /day. Both groups were fairly well balanced, with $2 / 3$ having $\mathrm{HCV}$ etiology and portal vein tumor thrombosis and one quarter showing extrahepatic disease. At 1 year, OS was $47 \%$ in the combination group and clearly not superior to the $58 \%$ in the sorafenib monotherapy group. The disease control rate similar with $45 \%$ in both groups. The median survival was statistically not different between the groups due to the small sample size but the 10 months median survival in the HAIC-combination group do not look good both in absolute terms and relative to the 15.2 months in the sorafenib monotherapy treatment arm. Considering the favorable baseline characteristics with only $50 \%$ BCLC C patients, close to $90 \%$ Child-Pugh A cirrhosis, and only $25 \%$ extrahepatic disease, the OS-data particularly in the HAIC-combination treatment group look disappointing, even when considering the relatively high rate of malignant portal vein thrombosis. Subgroup analysis also did not show any advantage for the HAIC-combination group. In patients with elevated AFP $\geq 400 \mathrm{ng} / \mathrm{mL}$, subgroup analysis even revealed a significant survival advantage for patients in the sorafenib monotherapy group. These differences could partly be ascribed to the differences in the post-study treatments, which were more common in the monotherapy group. But even this could be a consequence of the initial study treatment: despite similar adverse events in both groups, $23 \%$ of patients in the HAIC-combination group were not able to receive any further treatment after HAIC due to the deterioration in their performance status, raising serious questions about the safety of HAIC even in HCCpatients with good baseline parameters. Confirming the negative results of this prospective randomized phase-2 trial, the SILIUS multicenter randomized phase- 3 trial testing the combination of a different HAIC-regimen (lowdose cisplatin-fluorouracil) with sorafenib vs. sorafenib in Japanese advanced stage HCC patients reported negative results as well (10).

Despite the limitations of the SCOOP-2 trial with lower than expected patient inclusion due to recruiting problems, a relatively high number of patients unable to receive a full course of the planned treatment and the comparatively low median sorafenib-dose used, it seems as if HAIC should not be used as standard treatment for advanced stage HCC any longer. Not only is the cumulative evidence from prospective RCTs overwhelmingly against it. But with now four or more lines of systemic treatment available for these patients with proven survival benefit, we have a large number of options at hand and do not need to rely on treatments with negative trial results. Since the current treatment options can offer our patients an OS far beyond what has been reported in the SCOOP-2 trial (11) and using several lines of treatment in patients with good performance status confers additional survival benefit (12), avoiding treatments with relevant potential to do harm to our patients' liver function and general performance status becomes a priority. Instead of calling for more and larger trials of HAIC-combination therapy approaches, we should accept that conventional chemotherapy in any form for the treatment of advanced stage HCC looks like a dead-end street without proven efficacy alone or in combination with targeted agents and considerable detrimental impact on the patients' ability to receive further lines of therapy. It is probably time to move on.

\section{Acknowledgments}

Funding: None.

\section{Footnote}

Provenance and Peer Review: This article was commissioned by the editorial office, Annals of Translational Medicine. The article did not undergo external peer review.

Conflicts of Interest: All authors have completed the ICMJE uniform disclosure form (available at http://dx.doi. org/10.21037/atm-2020-96). MPR reports grants, personal fees and non-financial support from Bayer Healthcare, personal fees from Eisai, personal fees from Ipsen, personal fees from Roche, personal fees from BMS, personal fees and non-financial support from Lilly, outside the submitted work. The other authors have no conflicts of interest to declare.

Ethical Statement: The authors are accountable for all aspects of the work in ensuring that questions related 
to the accuracy or integrity of any part of the work are appropriately investigated and resolved.

Open Access Statement: This is an Open Access article distributed in accordance with the Creative Commons Attribution-NonCommercial-NoDerivs 4.0 International License (CC BY-NC-ND 4.0), which permits the noncommercial replication and distribution of the article with the strict proviso that no changes or edits are made and the original work is properly cited (including links to both the formal publication through the relevant DOI and the license). See: https://creativecommons.org/licenses/by-nc-nd/4.0/.

\section{References}

1. European Association for the Study of the Liver. EASL Clinical Practice Guidelines: management of hepatocellular carcinoma. J Hepatol 2018;69:182-236.

2. Vogel A, Cervantes A, Chau I, et al. Hepatocellular carcinoma: ESMO Clinical Practice Guidelines for diagnosis, treatment and follow-up. Ann Oncol 2018;29:iv238-55.

3. Kudo M, Matsui O, Izumi N, et al. JSH ConsensusBased Clinical Practice Guidelines for the Management of Hepatocellular Carcinoma: 2014 Update by the Liver Cancer Study Group of Japan. Liver Cancer 2014;3:458-68.

4. Finn RS, Zhu AX, Farah W, et al. Therapies for advanced stage hepatocellular carcinoma with macrovascular invasion or metastatic disease: A systematic review and meta-analysis. Hepatology 2018;67:422-35.

5. Ueda H, Fukuchi H, Tanaka C. Toxicity and efficacy of hepatic arterial infusion chemotherapy for advanced hepatocellular carcinoma (Review). Oncol Lett 2012;3:259-63.

Cite this article as: Peck-Radosavljevic M, Bota S, Hucke F. Time to stop using hepatic arterial infusion chemotherapy (HAIC) for advanced hepatocellular carcinoma? - the SCOOP-2 trial experience. Ann Transl Med 2020;8(21):1340. doi: 10.21037/atm-2020-96
6. Abou-Alfa GK, Johnson P, Knox JJ, et al. Doxorubicin plus sorafenib vs doxorubicin alone in patients with advanced hepatocellular carcinoma: a randomized trial. JAMA 2010;304:2154-60.

7. Llovet JM, Ricci S, Mazzaferro V, et al. Sorafenib in advanced hepatocellular carcinoma. $\mathrm{N}$ Engl J Med 2008;359:378-90.

8. Abou-Alfa GK, Shi Q, Knox JJ, et al. Assessment of treatment with sorafenib plus doxorubicin vs sorafenib alone in patients with advanced hepatocellular carcinoma: phase 3 CALGB 80802 randomized clinical trial. JAMA Oncol 2019;5:1582-8.

9. Kondo M, Morimoto M, Kobayashi S, et al. Randomized, phase II trial of sequential hepatic arterial infusion chemotherapy and sorafenib versus sorafenib alone as initial therapy for advanced hepatocellular carcinoma: SCOOP-2 trial. BMC Cancer 2019;19:954.

10. Kudo M, Ueshima K, Yokosuka O, et al. Sorafenib plus low-dose cisplatin and fluorouracil hepatic arterial infusion chemotherapy versus sorafenib alone in patients with advanced hepatocellular carcinoma (SILIUS): a randomised, open label, phase 3 trial. Lancet Gastroenterol Hepatol 2018;3:424-32.

11. Cheng AL, Qin S, Ikeda M, et al. IMbrave150: Efficacy and safety results from a ph III study evaluating atezolizumab (atezo) + bevacizumab (bev) vs sorafenib (Sor) as first treatment (tx) for patients (pts) with unresectable hepatocellular carcinoma (HCC). Ann Oncol 2019;30:ix186-7.

12. Finn RS, Merle P, Granito A, et al. Outcomes of sequential treatment with sorafenib followed by regorafenib for HCC: Additional analyses from the phase III RESORCE trial. J Hepatol 2018;69:353-8. 